Check for updates

Cite this: Phys. Chem. Chem. Phys., 2017, 19, 10562

Received 13th January 2017, Accepted 24th March 2017

DOI: $10.1039 / c 7 c p 00286 f$

rsc.li/pccp

\title{
Phonons spreading from laser-heated gold nanoparticle array accelerate diffusion of excitons in an underlying polythiophene thin film $\dagger$
}

\author{
David Rais, (D) * Miroslav Menšík, Bartosz Paruzel, Dharmalingam Kurunthuł and \\ Jiři Pfleger
}

\begin{abstract}
Localized surface plasmon (LSP) photophysical phenomena occurring in metal nanostructures are often presented as a method to effectively couple light into photovoltaic devices of sub-wavelength-scale thickness. However, the excitation of LSP is also associated with rapid energy dissipation leading to local heating, which affects the excitation energy pathway. We studied a system consisting of a planar gold nanoparticle (AuNP) array deposited at the surface of a semiconducting polymer thin film (P3HT). We observed heat transfer from laser pulse excited AuNPs into the P3HT, which was evidenced as a long-living thermochromic effect on transient optical absorption. By modeling of the ultrafast kinetics of exciton population evolution, we determined that their decay was caused by their mutual annihilation. The decay rate was controlled by a phonon-assisted one-dimensional diffusion mechanism with a diffusion constant of $2.2 \mathrm{~nm}^{2} \mathrm{ps}^{-1}$. The transferred heat resulted in an increase of the diffusion constant by a factor of almost 2, compared to the control system of P3HT without AuNPs. These results are of practical use for the design of plasmon-enhanced optoelectronic devices.
\end{abstract}

\section{Introduction}

The possibility of sub-wavelength manipulation of the optical field using localized surface plasmon (LSP) ${ }^{1-3}$ phenomena occurring at noble metal nanoparticles (NPs) is attractive for the design of various optoelectronic devices. The LSP phenomena are proposed as a mechanism for enhanced functionality in photovoltaics (PV), ${ }^{4}$ and light sources. ${ }^{5,6}$ For example, Rand et al. observed $^{7}$ higher power conversion efficiency (PCE) in a tandem organic PV cell, when an ultrathin metal cluster layer was placed at an interface of two individual sub-cells. The authors claimed that LSPs are, in part, responsible for the efficiency enhancement. It is, however, very difficult to unequivocally identify the nature of the PCE enhancement in a solar cell, since various competing phenomena occur due to the presence of metal NPs in the device.

Institute of Macromolecular Chemistry, AS CR, v.v.i., Heyrovského nám. 2,

16206 Prague, Czech Republic. E-mail: rais@imc.cas.cz

$\dagger$ Electronic supplementary information (ESI) available: The sample topology scheme; transient absorption data manipulation; results of the target analysis of the TA data; details of the spectral analysis of the TA data; detailed discussion of the TA spectra of AuNP@PS and neat P3HT systems; a comparison of the longliving TA feature in AuNP@P3HT with a thermochromic effect; dependence of GSB decay kinetics on the total energy in the pump pulse. See DOI: 10.1039/ c7cp00286f

\$ Present address: Department of Physics, Chemistry and Biology (IFM)/ Biomolecular and Organic Electronics (BIORG), Linköping University, SE-581 83 Linköping, Sweden.
These often lead to deterioration of the device performance due to effects such as exciton quenching at the metal surface, ${ }^{8,9}$ or LSP resonance absorption, which leads to rapid energy dissipation. ${ }^{10}$

Recently, Jägeler-Hoheisel et $a .^{11}$ carefully varied parameters of the Ag-NP array and its position in a PV device in an attempt to separate various interaction mechanisms that could lead to the observed overall enhancement of the PCE. They have found that the distance between the absorber layer and the Ag-NP layer is a key factor, even determining whether there is an enhancement, or degradation of the device performance. The spectrally resolved study of the external quantum efficiency enhancement indicated that it was the optical field distribution within the cell stack, which most prominently determined the observed enhancement/degradation effect. According to the simulation performed by Lindquist, et $a .^{12}$ the metal electrode surface parallel to the Ag-NP array may enhance the axial component of the time-averaged electric field. They observed an enhancement of PCE in their PV devices.

In this work, we investigated the mutual interaction between a planar array of gold nanoparticles (AuNP) and a thin film of poly(3-hexylthiophene) (P3HT) by means of ultrafast transient absorption (TA) spectroscopy. We have designed the architecture of the samples, in which the organic semiconductor is not located in the "hot spots" of the LSP resonance between the neighboring NPs in the array, but in the region of optical field depletion. It allowed us to avoid the plasmonic enhancement of 
the excited state photo-generation. We examined an impact of heat transfer from the laser-excited AuNP array on the ultrafast evolution of the excited states within the semiconducting polythiophene thin film. Since it may also play a role in the functionality of optoelectronic devices, this knowledge contributes to understanding the complexity of plasmon-related effects.

\section{Experimental}

\section{Sample preparation}

Poly(3-hexylthiophene), P3HT, $M_{\mathrm{w}}=44000$, regioregularity $96 \%$, obtained from LISICON, polystyrene, PS, $M_{\mathrm{w}}=50000$ and solvents (chloroform 99.9\%, toluene 99.9\% and dichlorobenzene 99\%) of CHROMASOLV HPLC purity grade obtained from Sigma Aldrich, were used as received. As a sample substrate, ultra-flat glass slides coated with a $20 \mathrm{~nm}$ layer of synthetic quartz (Ossila Ltd) were used.

Thin polymer films were deposited on the substrates (on the quartz-coated side) using the spin-casting method (3000 rpm for $30 \mathrm{~s}$ ) from chloroform (P3HT, concentration $0.25 \mathrm{wt} \%$ ), dichlorobenzene (P3HT, concentration $0.25 \mathrm{wt} \%$ ) and toluene (PS, concentration $1 \mathrm{wt} \%$ ) solutions. Then, a part of the thin polymer coating was washed off by dipping the edge of the substrate few times perpendicularly into the respective solvent. This treatment provided a clean area for reference measurements using the same substrate. The resulting polymer film thickness was $10 \mathrm{~nm}$, determined by scanning the height profile across the scratch in the polymer layer using a surface profilometer KLA-TENCOR P10. Subsequently, a layer of Au was deposited by physical vapor deposition (PVD, at pressure $2 \times 10^{-5}$ Torr, with deposition rate $0.1 \mathrm{~nm} \mathrm{~s}^{-1}$ ) on top of each substrate through a shadow mask covering one half of it. The effective thickness of the deposited $\mathrm{Au}$ layer $(1 \mathrm{~nm})$ as well as the deposition rate was determined using a crystal balance monitor. The samples of the neat P3HT film and the AuNP@P3HT composite were then annealed at $140{ }^{\circ} \mathrm{C}$ on a hot plate and encapsulated under a $\mathrm{N}_{2}$ atmosphere in a glovebox (60 ppm $\mathrm{O}_{2}, 2 \mathrm{ppm}$ moisture) with a microscope cover slip separated from the substrate with a $50 \mu \mathrm{m}$ gap (using a pair of optical fibers), and sealed at the edges with an epoxy resin.

\section{Characterization}

UV-Vis steady state spectra were recorded using a custom built double-channel fiber optics spectrophotometer based on a deuterium and halogen lamp light source (model DH2000, Ocean Optics) and two CCD-array spectrometers coupled to a common A/D converter (model ADC 1000USB, Ocean Optics). The light from the source was collimated and directed to the sample perpendicularly to its surface.

A pump-probe transient absorption (TA) spectrometer HELIOS (Ultrafast Systems LLC) was used for the time resolved absorption spectroscopy measurements. The details of the systems are described in our previous report, ${ }^{13}$ and are also specified in the section Transient absorption spectroscopy in the ESI. $\dagger$ Contrary to the previous report, we used pump pulses with linearly polarized light of a $550 \mathrm{~nm}$ central wavelength in the present work. The broadband linearly-polarized probe pulses contained a continuum of photon energies in two spectral ranges 400-800 $\mathrm{nm}$ and 850-1200 nm, depending on the configuration of white-light continuum generation. The mutual orientation of the electric field vectors of the pump and probe pulses in the sample was set at the magic angle $\left(54.7^{\circ}\right)$ in order to eliminate any contribution of the transition moment rotational diffusion to the decay kinetics. ${ }^{14}$ The energy of the pump pulses was set using a reflective neutral density filter, which could be varied continuously.

\section{Results}

\section{Steady state optical absorption study}

In Fig. 1, the spectra of AuNP arrays deposited on thin P3HT and PS films (designated as AuNP@P3HT and AuNP@PS, respectively) are compared to the spectra of the bare P3HT and PS films (without AuNP array, designated as neat P3HT and PS, respectively).

In our previous study, ${ }^{15}$ we described the structure of the AuNP array deposited by PVD on top of the thin films of these two polymer materials using transmission electron microscopy. We have found that the as-deposited AuNP@PS film had very closely matching NP size distribution and topological organization to the AuNP@P3HT film. For AuNPs on top of the P3HT thin film, the mean gold particle size was $d=3.4 \mathrm{~nm}$ with the standard deviation $\sigma=1.2 \mathrm{~nm}$; for PS the parameters of the AuNP array were $d=4.2$ and $\sigma=1.6 \mathrm{~nm}$. Since the PVD process ensured the same mean effective thickness of the AuNP layer (1 $\mathrm{nm}$ thick layer of gold), the slightly larger particles of the AuNP array deposited on PS, as seen by TEM, resulted in slightly larger interparticle distances.

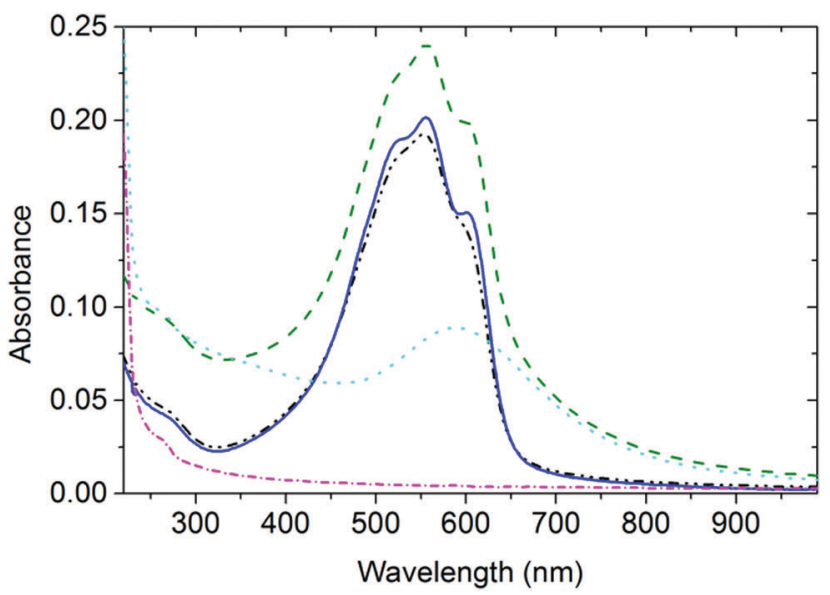

Fig. 1 Steady state optical absorption spectrum of the thin P3HT film after spin-casting followed by drying at room temperature (black dash-dot-dot line), after further annealing at $140{ }^{\circ} \mathrm{C}$ for $30 \mathrm{~min}$ in an inert atmosphere (blue solid line), and after deposition of the AuNP array (AuNP@P3HT sample, green dash line). The polystyrene thin film spectra before AuNP array deposition (neat PS, magenta dash-dot line) and after AuNP array deposition (AuNPaPS sample, cyan dotted, line). 


\section{AuNP array on polystyrene}

The optical absorption spectrum of the AuNP@PS system (Fig. 1) shows a broad band centered at around $590 \mathrm{~nm}$, and a small band at around $260 \mathrm{~nm}$. UV absorption in the AuNP@PS system is apparently caused by the phenyl groups of the PS film and can also be seen in the neat PS film. There is also strong contribution of Rayleigh scattering, with its characteristic wavelength dependence of $\sim \lambda^{4}$. Due to the lack of light absorption at wavelengths above $300 \mathrm{~nm}$ in the neat PS film (Fig. 1), the absorption spectrum of the AuNP@PS sample with the maximum at $590 \mathrm{~nm}$ is obviously dominated by the LSP resonance absorption (plasmon extinction) of the AuNP array. The mean diameter of the NPs deposited on PS was $d \approx 4.2 \mathrm{~nm}$, as obtained from the TEM studies reported in our previous paper. ${ }^{15}$ If the AuNPs with this diameter would be dispersed in dilute water colloid, it would show the plasmon extinction centered within the wavelength range $520-530 \mathrm{~nm} \cdot{ }^{16,17}$ The red-shifted maximum of the plasmon extinction band of about $70 \mathrm{~nm}$ indicates strong optical coupling of individual nanoparticles in the AuNP array. ${ }^{18-20}$

\section{Interaction between the AuNP array and the P3HT film}

The resulting spectrum of the P3HT thin film after annealing could be fitted with a sum of one broad Gaussian function centered at
$383 \mathrm{~nm}$ corresponding to the optical transitions in amorphous coils, and three Gaussians centered at 537, 563, and $610 \mathrm{~nm}$, corresponding to the $0-2,0-1$, and $0-0$ vibronic transitions, respectively, of the P3HT chains in crystalline domains. ${ }^{21,22}$ We could clearly recognize the vibronic structure of $\mathrm{P} 3 \mathrm{HT}$ also in the spectrum of AuNP@P3HT samples ( $c f$. red and green curves in Fig. 1). It means that the structural order in the $\mathrm{P} 3 \mathrm{HT}$ film has been mostly preserved after PVD deposition of the AuNP array on top of it.

\section{Time-resolved spectroscopic study}

For better understanding the photophysical interaction between the AuNP array and the underlying P3HT film in the AuNP@P3HT system we studied the time evolution of the optical absorption of the AuNP@P3HT system using TA spectroscopy. We will first turn our attention to the photoinduced TA evolutions in the neat thin P3HT film and in the AuNP@PS system and use the results to estimate the contribution of AuNP array to the transient kinetics of the excited states of P3HT in the AuNP@P3HT system.

\section{The neat P3HT thin film}

The representative TA spectra of the neat P3HT, and AuNP@ P3HT films together with the TA spectrum of the AuNP@PS
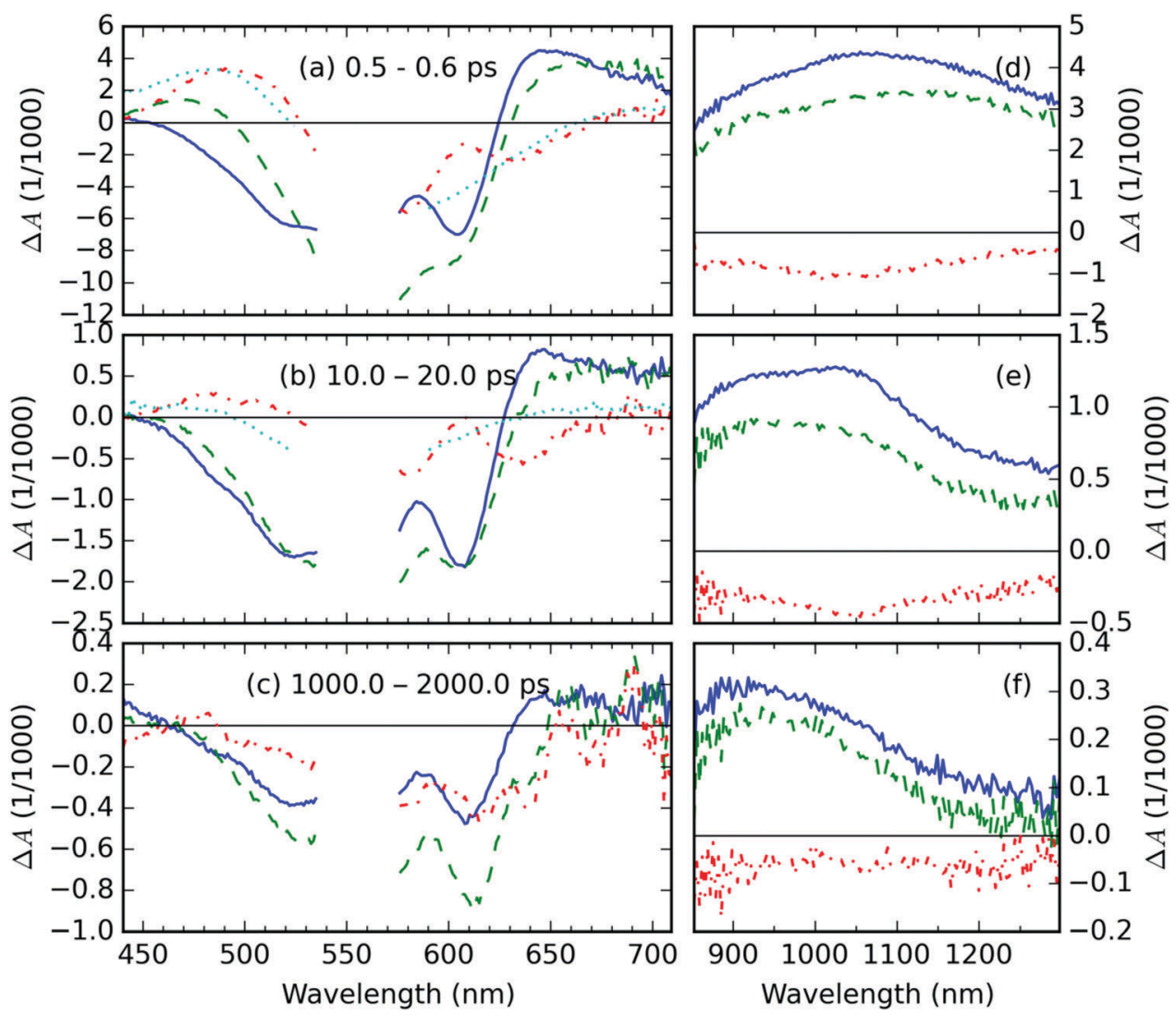

Fig. 2 TA spectra of P3HT films averaged over selected delay time intervals (indicated in the legends). The color indicates the type of sample: difference (red dash-dot line) between the AuNP@P3HT composite (green dashed line) and the neat P3HT (blue solid line). The TA spectrum of AuNP@PS is shown as cyan dotted lines in panels ( $a$ and b) for comparison. The left $(a-c)$ and right $(d-f)$ panels in each row correspond to Vis- and NIR-probe data, respectively; the delay time interval is the same for both panels in each row. The data in the spectral range of 535-575 nm were removed due to the high content of scattered light of pump pulses. 
system are shown in Fig. 2. The corresponding kinetic traces are shown in Fig. 3. We observed a well-resolved negative signal composed of a broad band spanning from 450 to $580 \mathrm{~nm}$ and a rather sharp band centered at $605 \mathrm{~nm}$. This part of the spectrum overlaps with the steady state absorbance band in the neat P3HT film, particularly the peak at $605 \mathrm{~nm}$ corresponds to the $0-0$ vibronic transition within the crystal domains. Therefore, both negative TA features can be understood as a measure of photoinduced depletion of the ground state (denoted $\mathrm{S}_{0}$ ), i.e. ground state bleaching (GSB). This observation and interpretation agree well with previous reports of the time-resolved spectroscopic studies of P3HT thin films. ${ }^{22-24}$

We further observed a positive TA feature generated immediately with laser excitation, at around $1150 \mathrm{~nm}$ (in Fig. 2d). Another positive early TA signal emerges at around $650 \mathrm{~nm}$ (Fig. 2a). The respective kinetic traces (Fig. 3c and e) show a slight delay of $\approx 200 \mathrm{fs}$ in the position of the maximum of TA at $650 \mathrm{~nm}$, which, respectively, suggests that the features at 650 and $1150 \mathrm{~nm}$ belong to different photochemical species. In agreement with Guo, et al. ${ }^{23}$ and Ogata, et al. ${ }^{24}$ we adopted the interpretation of those species as polaron pairs (PP) and

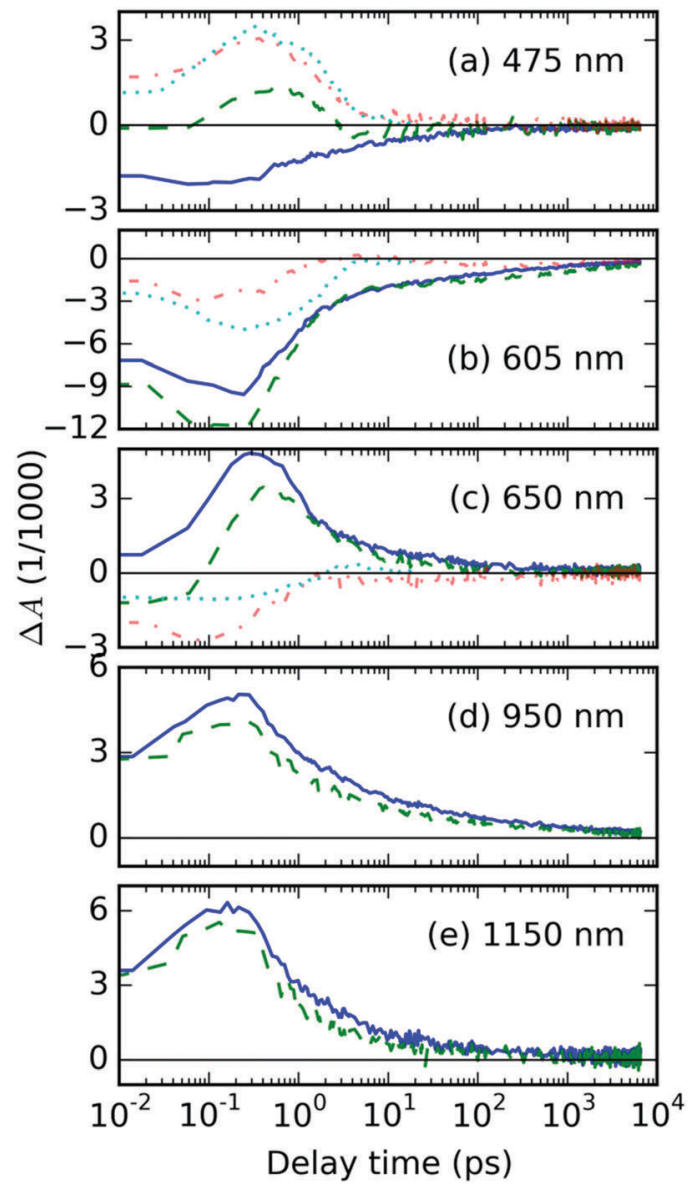

Fig. 3 The TA kinetics traces of the studied systems at the indicated probe wavelengths. The color indicates the type of sample: difference (red dash-dot line) between the AuNP@P3HT composite (green dashed line) and the neat P3HT (blue solid line). The cyan dotted lines show TA kinetics recorded in the AuNP@PS composite, for comparison. singlet excitons $\left(\mathrm{S}_{1}\right)$, respectively. Accordingly, we attribute the long-living feature at $950 \mathrm{~nm}$, which becomes prevalent in NIR probe spectra on 10 ps timescale (see Fig. 2e), to free polarons (FP).

\section{The AuNP@PS composite}

The TA spectra of the AuNP@PS sample deposited on a glass substrate are shown in Fig. 2; the respective kinetic traces are shown in Fig. 3. Since there is no overlap of the optical absorption of polystyrene and AuNP array (see Fig. 1), the direct photoexcitation of the supporting PS thin film was avoided. The TA spectrotemporal evolution in the composite following the excitation by femtosecond (fs) laser pulses with a central wavelength of $550 \mathrm{~nm}$ is thus exclusively determined by the AuNP array.

The TA signal of the AuNP@PS system (see the cyan curve in Fig. 2a) shows three well-resolved spectral regions: the negative signal in the probe wavelength range $520-650 \mathrm{~nm}$ and positive bands in the intervals of 400-520 $\mathrm{nm}$ and $650-800 \mathrm{~nm}$. The TA signal decay kinetics in both positive and negative parts of the spectra was simultaneous. Using the global analysis approach, ${ }^{25-27}$ we could identify 3 exponential decay times $\tau_{\mathrm{i}}: 0.57,1.17$ and $9.9 \mathrm{ps}$ (see Fig. S3a and b in the ESI $\dagger$ ).

The photophysical behavior of noble metal nanostructures under pulsed excitation was summarized in a comprehensive review by Hartland. ${ }^{17}$ The TA signal evolution shown in Fig. 2 and 3 can be understood in the following way: as the absorbed pump pulse induced the ultrafast heating of the electron gas, the absorption of AuNP array broadened and simultaneously lowered its amplitude, which resulted in the observed negative $\Delta A$ in the middle of two positive spectral lobes, even at the shortest delay times ( $c f$. Fig. 2a). This effect of the elevated temperatures was reported previously by Doremus for gold nanoparticle dispersions in glass. ${ }^{16}$ He showed that the peak amplitude of the steady-state absorption band drops linearly by $30 \%$ in the temperature range from -200 to $500{ }^{\circ} \mathrm{C}$, i.e. by $4.29 \%$ per $100 \mathrm{~K}$. Following this similarity and considering the trace of $\Delta A_{\text {AuNP@PS }}$ at a probe wavelength of $605 \mathrm{~nm}$ (Fig. 3b) and the peak of the steady state absorbance at $600 \mathrm{~nm} A_{\text {AuNP@PS }}=0.08$ (blue curve in Fig. 1), we can estimate the increase of the electron gas temperature in the AuNP array to be about $160 \mathrm{~K}$ above room temperature. About $4 \mathrm{ps}$ after photoexcitation the equilibrium temperature with the gold lattice was reached at about $9 \mathrm{~K}$ above room temperature. We identified the lifetime of $1.17 \mathrm{ps}$ as the time needed for cooling the hot electron gas by electron-phonon coupling. During this process, part of the energy is redistributed to surface phonon modes of the AuNPs, allowing the heat exchange between AuNPs and the supporting PS film.

\section{The effects of AuNP array on the P3HT thin film}

Here, we will analyze the temporal evolution of TA spectra of the AuNP@P3HT thin film, focusing on the influence of the AuNP array. Fig. 2 and 3 show the representative TA spectra and kinetic traces, following photoexcitation by fs laser pulses with a central wavelength of $550 \mathrm{~nm}$. In both figures, we show also 
the residual signal after subtraction of the signal of the neat P3HT film from the signal of AuNP@P3HT:

$$
\Delta A_{\text {resid }}(t, \lambda)=\Delta A_{\text {AuNP@Р3нт }}(t, \lambda)-\Delta A_{\mathrm{P} 3 \mathrm{HT}}(t, \lambda) .
$$

The $\Delta A_{\text {resid }}(t, \lambda)$ spectra are compared to the spectra of P3HT and of the AuNP@PS system, measured using the same excitation pulse conditions (i.e. its spectral and temporal envelope and energy).

The residual TA spectrum, $\Delta A_{\text {resid }}$ at the sub-picosecond delay time after excitation, shows a broad and structured negative band in the probe region of 490-630 nm, surrounded by positive bands on both sides (red curve, Fig. 2a). From the overlap of the residual signal $\Delta A_{\text {resid }}(t, \lambda)$ with the TA spectra of the AuNP@PS composite (cyan curve, Fig. 2a), we can deduce that the positive signal at the short probe wavelengths originates from the excited electron gas in the AuNP array. This becomes more evident from the detailed kinetic traces of the TA signal at a $475 \mathrm{~nm}$ probe wavelength (Fig. 3a).

In the region, where negative $\Delta A_{\text {resid }}(t, \lambda)$ is negative, we could see overlapping negative signals from the AuNP array and from the underlying P3HT film; the contribution from the latter apparently results in a structured shape of $\Delta A_{\text {resid }}(t, \lambda)$. The residual spectrum clearly shows the same structuring, but in the opposite direction. This suggests that in the calculation of $\Delta A_{\text {resid }}(t, \lambda)$ using eqn (1), the contribution of the GSB of the P3HT film has been overestimated. This effect probably arises from the combined effects of (i) absorption of pump light in the AuNP array - about 20\% intensity loss, and (ii) the near-field (plasmonic) effect. The nearfield effect creates regions of field depletion on either side of the array, together with hot-spots of field enhancement in the interparticle gaps. In our case, the P3HT is located in the area of nearfield depletion, similarly to the system presented in ref. 28.

The two broad positive bands in the 630-700 nm and 900$1300 \mathrm{~nm}$ regions overlap well with the spectra of PP and of singlet excitons $\left(\mathrm{S}_{1}\right)$ in the neat P3HT film. Due to the similarity in the shape and position of the TA features in the AuNP@P3HT composite and neat P3HT thin films, we can use the same assignment of these bands as in the previous section focused on the neat P3HT film.

\section{Laser induced heating}

In order to explain the apparent extension of the lifetime of the TA signal recorded at $605 \mathrm{~nm}$ due to the presence of the AuNP array ( $c f$. green and blue curves in Fig. 3b), we invoke laserinduced heating artifacts in the TA experiments discussed in the literature by Rao, et $a .^{29}$ The authors of the cited work identified a laser pump-induced heating artifact in the TA spectra of pentacene. These were actually artifacts originating in the thermal modulation caused by the laser pump, due to thermochromism of this material. This phenomenon is spectrally located in the GSB area, due to the fact that it modulates optical transitions from the ground state, and it has lifetime in the order of 100 ns. In Fig. S6 in the ESI, $\dagger$ we show that it indeed overlaps quite well with the residual TA signal $\Delta A_{\mathrm{AuNP} @ \mathrm{P} 3 \mathrm{HT}}-\Delta A_{\mathrm{P} 3 \mathrm{HT}}$ averaged over the delay time interval 1-2 ns, as presented in Fig. 2c.
Nevertheless, the presence of the AuNP array increases the intensity of the long-living TA signal by almost a factor of 2 . When we compare this figure with the situation of the TA signal in the wavelength range of FP states (950 nm probe, see Fig. 3d), we can see that the ratio is opposite, i.e. the signal in the composite with AuNP array is lower. Similar finding was obtained also for the other excited states: $S_{1}$ states at $1150 \mathrm{~nm}$ as well as PP states at $650 \mathrm{~nm}$ show a weaker signal in the AuNP@P3HT composite than in the neat P3HT. This could only mean that there is another source of the TA signal in the GSB region.

The existence of another electronic excited state is unlikely since no other transient absorption bands were found in the relatively large spectroscopic bandwidth of our experimental setup. On the other hand, the above mentioned thermal effect deserves a more detailed discussion. The total energetic input can be deduced from the ratio of the optical absorption maxima of the two systems ( $c f$. Fig. 1): the amount of extra energy stored and dissipated in the AuNP@P3HT system is roughly higher by $7 \%$ than in the neat P3HT. According to the literature the heat capacity in bulk P3HT was estimated ${ }^{30}$ as $C \approx 10^{6} \mathrm{~J} \mathrm{~m}^{-3} \mathrm{~K}^{-1}$, while the thermal conductivity was ${ }^{31} K \approx 0.5-2.29 \mathrm{~W} \mathrm{~K}^{-1} \mathrm{~m}^{-1}$. Therefore we estimate the heat diffusion constant in the bulk P3HT as $D=C / K \approx 10^{-6} \mathrm{~m}^{2} \mathrm{~s}^{-1}$, which corresponds to the diffusion length of $1 \mathrm{~nm}$ for $1 \mathrm{ps}$, and $c a .3 .3 \mathrm{~nm}$ for $10 \mathrm{ps}$. However, due to the crystalline-like structure of domains in the P3HT thin films, a faster quasi 1-D heat transfer could by promoted at shorter distances. It was found that acoustic phonons spread with velocity $c=2.8 \mathrm{~nm} \mathrm{ps}{ }^{-1} \cdot{ }^{32}$ It means that the heat transfer rate is fast enough to induce the thermal (thermochromic) TA artifact at the ns time scales in question. This, together with the spectral overlap of the GSB signal with the thermochromic effect due to laser irradiation (shown in Fig. S6 in the ESI $\dagger$ ), leads us to the following explanation: the negative TA signal in the spectral region around $600 \mathrm{~nm}$ in AuNP@P3HT contains strong contribution from the phenomenon of thermochromism in the P3HT film caused by heat transfer from the laser-heated AuNP array.

\section{Singlet exciton recombination}

To gain more insight into the evolution of the excited states and their mutual relationships in the studied systems we attempted to analyze the TA spectra using the target analysis. ${ }^{25,26,33}$ We modeled the excited state evolutions in neat P3HT films by a sequential unbranched 5-compartment model. In the case of the AuNP@P3HT composite, we added a separate branch with a single lifetime to model the evolution of the plasmon resonance contribution. The details are described in the $\mathrm{ESI} \dagger$ in the section Target model analysis of the vis-WLC transient absorption data. In summary, the analysis required at least 5 compartments for a good fit, each associated with a one species associated differential spectrum (SADS) and one lifetime. However, the resulting shapes of SADSs were very similar in the broad range of their respective lifetimes. This suggests that the system of linear differential equations used in the target analysis did not reflect the actual photochemical reality in the systems, i.e. most of the SADS contained contributions 
from all the photochemical species ( $\mathrm{S}_{1}, \mathrm{FP}$ and PP). As we will show below, the presence of mutual collisions between the (mobile) excited states could be a possible explanation of the multi-exponential kinetics of the excited state evolution.

For the analysis of the kinetics in the NIR region we chose the spectral analysis approach inspired by the work of Ogata et $a l^{24}$ It allowed us to project the spectrotemporal evolution into temporal evolution of weight parameters of the time invariant spectral features, without a priori knowledge of the model kinetic equation. The assumption of the time invariance of the spectral profiles is sound if the relevant processes contributing to the kinetics occur on a time-scale longer than vibrational relaxation processes associated with the reorganization energy of P3HT. Formally, the spectral model function shapes may be influenced by the laser-induced heating described above. However, considering that our above estimate of the temperature increase was only $9 \mathrm{~K}$ in the AuNP@P3HT sample, we choose to neglect the effect of temperature on the spectral model functions. This also allowed us to optimize the spectral positions and widths globally (as common parameters) using TA spectra recorded at all delay times. This greatly reduced uncertainties in the parameter estimations.

Based on the spectral shapes and their attribution to the photochemical species discussed above, we approximated the spectrum of the TA signal for a given delay time by a linear combination of two spectral profiles with Gaussian distribution in energy scale. Our analysis provided globally optimized shapes and positions of Gaussian functions centered at $E_{\mathrm{S}}^{\text {center }}=(0.96 \pm$ $0.24) \mathrm{eV}$ and $E_{\mathrm{PP}}^{\text {center }}=(1.30 \pm 0.24) \mathrm{eV}$ with function half-widths, $\sigma_{\mathrm{S}}=(0.19 \pm 0.30) \mathrm{eV}$ and $\sigma_{\mathrm{FP}}=(0.16 \pm 0.1) \mathrm{eV}$, respectively. The confidence intervals are set at twice the standard deviations; see Table $\mathrm{S} 2$ in the ESI. $\dagger$

The amplitudes of these two functions, $C_{\mathrm{FP}}(t)$ and $C_{\mathrm{S}}(t)$, represented the relative contributions of the FP and the $S_{1}$ species to the overall TA signal intensity. Due to the Beer-Lambert law, we can use the $C_{\mathrm{FP}}$ and $C_{\mathrm{S}}$ parameters as measures of fractional population of the respective species.

A full description of the fitting results is given in the ESI, $\dagger$ section Spectral analysis of the NIR-probe TA data. In Fig. 4a, we show only the resulting evolutions of the concentration parameters $C_{\mathrm{FP}}(t)$ and $C_{\mathrm{S}}(t)$, each normalized to the global maximum of the respective curve.

In Fig. $4 \mathrm{a}$, one can see that $\mathrm{S}_{1}$ in both the neat P3HT and AuNP@P3HT thin films undergoes rapid decay, reaching 90\% population loss within approx. 11 and 6 ps, respectively. Both dependences appear linear in the double log scale of Fig. 4, which resembles a power law decay of the populations, with the same apparent slope $d\left(\log C_{\mathrm{S}}\right) / d(\log t)=-1 / 2$ in both systems. Such dependence is usually caused by mutual annihilation of excitons, ${ }^{34-36}$ which is a bi-molecular process modelled by a general kinetic equation in the form:

$$
\frac{\mathrm{d} C_{\mathrm{S}}}{\mathrm{d} t}=-\gamma(t) C_{\mathrm{S}}^{2}
$$

where $\gamma(t)$ is the (time-dependent) bimolecular rate parameter. Its explicit form is dependent on the mechanisms of interaction. Assuming a mutual annihilation of excitons Engel, et al. ${ }^{32}$ derived for the parameter $\gamma(t)$ an equation: ${ }^{34}$

$$
\gamma(t)=a t^{-1 / 2},
$$

where parameter $a$ is already time invariant. The authors of the cited ref. showed that this kind of dependence corresponds either to one-dimensional diffusion limited bimolecular recombination or to a single-step long range Förster-type annihilation. The latter mechanism requires a significant spectral overlap of the photoemission and excited state absorption of the $\mathrm{S}_{1}$ states and, simultaneously, depends on the transition dipole moments of both these processes as well. In the present work, we cannot support the Förster energy transfer mechanism due to a very weak photoluminescence signal (data not shown),
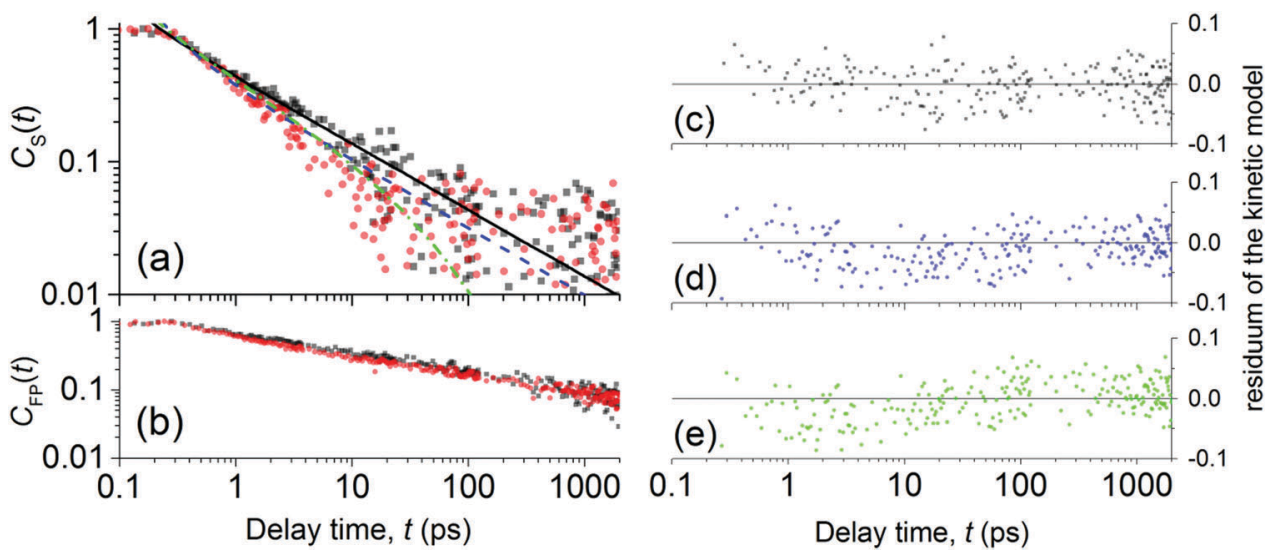

Fig. 4 Normalized evolutions of the concentration of (a) $S_{1}$ states and of (b) FP states obtained from the spectral analysis of NIR-probe TA spectra. Experimental data are denoted by full symbols: neat P3HT (black squares) and AuNP@P3HT composite (red circles). The best-fitted simulation of $C_{S}(t)$ decay due to exciton mutual annihilation (eqn (2)) in neat P3HT and AuNP(AP3HT are plotted in (a) with solid black and dashed blue lines, respectively. The residual plots of the simulations are plotted in (c) and (d), respectively. The green dash-dotted curve in (a) simulates the population decay in AuNP@P3HT, due to exciton mutual annihilation like in neat P3HT, with an additional decay channel controlled by exciton self-annihilation at the AuNP array surface (eqn (4)); the respective model residuals are in (e). 
indicating a weak transition dipole moment of the $\mathrm{S}_{1}$ state photoemission from the interchain excitons in the $\mathrm{H}$-aggregated polymer structure. ${ }^{37}$ On the other hand the significant $S_{1}$ state $1 \mathrm{D}$ diffusion model reflects well the structure of our samples, in which the $2 \mathrm{D}$ layered packing of P3HT chains constrained by boundaries of the thin film can locally promote the (quasi) 1D character of $\mathrm{S}_{1}$ state diffusion.

Analyzing the temporal evolutions $C_{\mathrm{S}}(t)$ in terms of the exciton annihilation model according to eqn (2) and (3), we found that its solution fits the population parameters $C_{\mathrm{S}}(t)$ in the case of a neat P3HT sample excellently up to the delay time $t \approx 2000$ ps (see the evenly distributed residuals Fig. 4c). In the case of the AuNP@P3HT sample, the agreement of this model with the (derived) experimental data is not so good, systematically deviating in a delay time range 3-50 ps. We found the best-fitting values of the parameter $a=1.15(\mathrm{ps})^{-\frac{1}{2}}$ and $a=1.6(\mathrm{ps})^{-\frac{1}{2}}$, for the neat P3HT and AuNP@P3HT systems, respectively. If our hypothesis of the diffusion model is correct, the different values of parameter $a$ in each of the systems indicate that the presence of the AuNP array in contact with the surface of the P3HT film increased the 1D diffusivity of $S_{1}$ excitons after photoexcitation.

In Fig. 4b, we show the evolution of the normalized concentration of free polarons, $C_{\mathrm{FP}}(t)$. We observed that differences in the kinetics of $C_{\mathrm{FP}}(t)$ decays for the neat P3HT and AuNP@P3HT are statistically less significant than differences in $C_{\mathrm{S}}(t)$ decays discussed above. In both cases, we observed an immediate increase of $C_{\mathrm{FP}}(t)$, followed by a steady power-law type decay. The decay appears to be slightly faster in the range 1-100 ps in the case of the AuNP@P3HT sample. Further discussion of the evolution of the concentration of FP states, as well as of PP states is beyond the scope of this report and will be discussed elsewhere.

The extra temperature rise due to the thermal energy transfer from the AuNP array in the AuNP@P3HT composite (vide supra) may also be responsible for the observed faster decay of $S_{1}$ states Fig. 4a: assuming a simple dispersion relation $\omega_{\text {vib }}=c k=2 \pi c / \lambda_{\text {vib }}$ for the eigenfrequency of the acoustic phonons we found that all those phonons satisfying $\hbar \omega_{\text {vib }}=2 \pi \hbar c / \lambda_{\text {vib }}<k_{\mathrm{B}} T$ can be thermally excited. Hence, at room temperature $T=300 \mathrm{~K}$ all acoustic phonons with wavelengths $\lambda_{\text {vib }} \geq 2 \pi \hbar c / k_{\mathrm{B}} T \equiv 0.46 \AA$ could be excited. On the other hand, there is an upper edge for $\lambda_{\text {vib }}$ in P3HT, given by the conjugation length, where the coherent phonon dynamics is destroyed. As the latter is significantly longer than $0.46 \AA$, there is a wide continuum of possible acoustic phonons, which can be thermally excited. For the increasing value of the local temperature $T$ of the P3HT layer, the probability of the thermal excitations of that manifold increases, resulting in a continuum of acoustic phonons flowing from the AuNPs. The eigenstates of the phonons as well as the delocalized excitons possess the Bloch wave ( $k$-dependent) structure so that their coupling promotes the coherent transfer of the wave packet along the chain. We conjecture that the acoustic phonons can increase the exciton diffusivity along the 1-D P3HT chain.

Previously, Szeremeta, et al. ${ }^{38}$ observed a decrease of the photoluminescence intensity upon the addition of metal NPs into a P3HT film. They hypothesized, that the metal (Ag) NPs can act as a recombination boundary for the diffusing excitons. In order to clarify whether this could be the case also in our AuNP@P3HT system, we tried to fit the experimental data with a modified model given in eqn (2) and (3), including an additional term for a monomolecular recombination of excitons: $:^{34}$

$$
\frac{\mathrm{d} C_{\mathrm{S}}}{\mathrm{d} t}=-\frac{a C_{\mathrm{S}}^{2}}{\sqrt{t}}-\frac{b C_{\mathrm{S}}}{\sqrt{t}}
$$

where parameter $b$ (proportional to the diffusion constant) is time independent. The kinetics of exciton decay is controlled by $1 \mathrm{D}$ diffusion with the initial condition $C_{\mathrm{S}}(x, t=0)=$ const. and the boundary condition $S(x=0, t)=0$ (ideal sink at the AuNP array; $x \in\langle 0, L\rangle$ is the spatial coordinate along the film thickness, $L)$. Here we fixed the value $a=1.15$ (ps) $)^{-\frac{1}{2}}$ found for the neat P3HT film, assuming that the AuNP array neither influences the diffusion of excitons nor their annihilation and that the only impact of the AuNP array is due to the recombination of excitons at the metallic boundary. We found that there was no value of $b$, which could explain the experimental decay curves in AuNP@P3HT better than the previous model with faster bimolecular annihilation of the excitons. The optimized value of $b=0.12(\mathrm{ps})^{-\frac{1}{2}}$ was found to fit well with the data only in long delay times - as shown in Fig. 4a and the residual plot in Fig. 4e.

Thus, we interpret these findings as the clear evidence that the dominant process in the $S_{1}$ population decay in the P3HT film is their mutual annihilation, and that the rate of their bimolecular interaction is increased significantly by the presence of the AuNP array. The presence of bimolecular reactions was additionally supported by the dependence of decay kinetics of the GSB signal on the total energy of the pump pulse, as we have shown in Fig. S7 in the ESI. $\dagger$

We have also shown that the AuNP array does not act as a sink for the $S_{1}$ states, at least in the early delay time range $(t<50 \mathrm{ps})$. Additionally, we conjecture that annihilation is controlled by $1 \mathrm{D}$ diffusion of $\mathrm{S}_{1}$ excitons through the $\mathrm{P} 3 \mathrm{HT}$ thin film and that the presence of the AuNP array increases their diffusivity after photoexcitation through a phonon-assisted motion.

The $1 \mathrm{D}$ exciton diffusion constant $D_{1 \mathrm{D}}$ can be obtained from the fitted normalized kinetics of exciton-exciton collision using the following equation: ${ }^{34}$

$$
a=\frac{n_{0}}{\bar{x} N_{0}} \sqrt{\frac{8 D_{1 \mathrm{D}}}{\pi}}
$$

where $\bar{x} \approx 0.4 \mathrm{~nm}$ corresponds to the inter-monomer distances and $N_{0} \approx 4(\mathrm{~nm})^{-3}$ is the mean monomer density in P3HT, according to a very recent work of Toman, et al. ${ }^{39}$ The initial excitation density $n_{0}$ created by the pump pulse in the probed area of the sample can be calculated according to ref. 36 as

$$
n_{0}=\frac{\lambda_{\text {pump }}}{h c} \cdot E_{\text {pump }}\left(1-\mathrm{e}^{-\frac{2 r_{\text {probe }}{ }^{2}}{r_{\text {pump }}^{2}}}\right) \frac{\left(1-10^{-\mathrm{OD}\left(\lambda_{\text {pump }}\right)}\right)}{\pi r_{\text {probe }^{2} L} L}
$$

where in our experiment the pump wavelength $\lambda_{\text {pump }}=550 \mathrm{~nm}$, the Gaussian radius of the pump pulse fluence in the focal plane $r_{\text {pump }}=270 \mu \mathrm{m}$, while that for the probe $r_{\text {probe }}=70 \mu \mathrm{m}$. 
The optical density of the neat P3HT sample at the pump wavelength was 0.18 . The pulse total energy was set to $E_{\text {pump }}=1 \mu \mathrm{J}$. The sample thickness was $L=10 \mathrm{~nm}$. For those values we found that the fraction of $c a$. 0.2 of the monomers was initially photoexcited. For the $1 \mathrm{D}$ exciton diffusion constant $D_{1 \mathrm{D}}$ we obtained an estimate $D_{1 \mathrm{D}} \approx 2.2 \mathrm{~nm}^{2} \mathrm{ps}^{-1}$ for the case of neat P3HT, while for the case of heated P3HT we obtained $D_{1 \mathrm{D}} \approx 4.3 \mathrm{~nm}^{2} \mathrm{ps}^{-1}$. Thus we have determined about a two-fold increase of the $1 \mathrm{D}$ exciton diffusion constant at the ps timescale due to the flow of phonons from the laser-heated AuNPs to the underlying P3HT film.

Recently, Tamai, et $a .^{40}$ also concluded that excitons in P3HT undergo 1D diffusion, and the published value of the diffusion constant which is lower by a factor of 2.7 from our estimate for the neat P3HT. But in their work they have used different forms of the bimolecular annihilation rate $\gamma(t)$ for the $1 \mathrm{D}$ diffusion problem, arising from different theoretical considerations. Moreover, the absolute value of $D_{1 \mathrm{D}}$ is difficult to compare, when determined from measurements under different experimental conditions, but the conclusion of the 1D nature of exciton diffusion appears to be consistent.

\section{Conclusions}

It was found that the TA of the AuNP@P3HT system is strongly affected by heating due to the absorption of laser pulse energy by AuNPs, followed by energy dissipation into P3HT. Particularly, the long-living TA signal of the AuNP@P3HT system in the spectral region of GSB was in part caused by a thermochromic effect, due to extra heating of the $\mathrm{P} 3 \mathrm{HT}$ film by heat transfer from the AuNP array.

Additionally, acoustic phonons emanating from the AuNP array increased singlet exciton diffusivity in the P3HT layer of the composite. It results in a reduced lifetime of excitons due to their accelerated mutual annihilation.

\section{Author contributions}

The manuscript was written through contributions of all authors. All authors have given approval to the final version of the manuscript.

\section{Abbreviations}

$\begin{array}{ll}\text { 1-D } & \text { One-dimension(al) } \\ \text { AuNP } & \text { Gold nanoparticle } \\ \text { FP } & \text { Free polaron } \\ \text { GSB } & \text { Ground-state bleaching } \\ \text { LSP } & \text { Localized surface plasmon } \\ M_{\mathrm{w}} & \text { Molecular weight (in daltons) } \\ \text { NP } & \text { Nanoparticle } \\ \text { OD } & \text { Optical density } \\ \text { P3HT } & \text { Poly(3-hexyl thiophene) } \\ \text { PCE } & \text { Power conversion efficiency } \\ \text { PP } & \text { Polaron pair }\end{array}$

PS

PV

PVD

$\mathrm{S}_{1}$

SADS

TA

TEM

UV

Vis

\section{Acknowledgements}

D. R. is thankful to his colleague Petr Toman for discussions of numerous theoretical models. The authors acknowledge financial support from the Ministry of Education, Youth and Sports of the Czech Republic within the National Sustainability Program I (NPU I, project POLYMAT LO1507) and with project LD14011. The support from the European Union (COST Action, MP1202) and from the Grant Agency of the Czech Republic (project GA15-05095S) is also acknowledged.

\section{References}

1 G. Mie, Ann. Phys., 1908, 330, 377-445.

2 C. F. Bohren and D. R. Huffman, Absorption and scattering of light by small particles, Wiley, New York, 1983.

3 H. C. van de Hulst, Light Scattering by Small Particles, Dover Verlag, 1981.

4 H. A. Atwater and A. Polman, Nat. Mater., 2010, 9, 205-213.

5 K. Leosson, J. Nanophotonics, 2012, 6, 061801.

6 P. Törmö and W. Barnes, Rep. Prog. Phys., 2015, 78, 013901.

7 B. P. Rand, P. Peumans and S. R. Forrest, J. Appl. Phys., 2004, 96, 7519-7526.

8 H. Becker, S. E. Burns and R. H. Friend, Phys. Rev. B: Condens. Matter Mater. Phys., 1997, 56, 1893-1905.

9 D. E. Markov and P. W. M. Blom, Phys. Rev. B: Condens. Matter Mater. Phys., 2005, 72, 161401.

10 G. Baffou and R. Quidant, Laser Photonics Rev., 2013, 7, 171-187.

11 T. Jägeler-Hoheisel, F. Selzer, M. Riede and K. Leo, J. Phys. Chem. C, 2014, 118, 15128-15135.

12 N. C. Lindquist, W. A. Luhman, S.-H. Oh and R. J. Holmes, Appl. Phys. Lett., 2008, 93, 123308.

13 D. Rais, P. Toman, J. Černý, M. Menšík and J. Pfleger, J. Phys. Chem. A, 2014, 118, 5419-5426.

14 G. Fleming, J. Morris and G. Robinson, Chem. Phys., 1976, 17, 91-100.

15 B. Paruzel, E. Pavlova, J. Pfleger, M. Šlouf, K. Halašová and r. Mikmeková, Chem. Pap., 2017, 71, 401-408.

16 R. H. Doremus, J. Chem. Phys., 1964, 40, 2389-2396.

17 G. V. Hartland, Chem. Rev., 2011, 111, 3858-3887.

18 I. Šloufová Srnová and B. Vlčková, Nano Lett., 2002, 2, 121-125.

19 K. Isozaki, T. Ochiai, T. Taguchi, K.-I. Nittoh and K. Miki, Appl. Phys. Lett., 2010, 97, 221101. 
20 M. P. Konrad, A. P. Doherty and S. E. J. Bell, Anal. Chem., 2013, 85, 6783-6789.

21 J. Clark, C. Silva, R. H. Friend and F. C. Spano, Phys. Rev. Lett., 2007, 98, 206406.

22 D. Herrmann, S. Niesar, C. Scharsich, A. Köhler, M. Stutzmann and E. Riedle, J. Am. Chem. Soc., 2011, 133, 18220-18233.

23 J. Guo, H. Ohkita, H. Benten and S. Ito, J. Am. Chem. Soc., 2009, 131, 16869-16880.

24 Y. Ogata, D. Kawaguchi and K. Tanaka, Sci. Rep., 2015, 5, 8436.

25 I. H. van Stokkum, D. S. Larsen and R. van Grondelle, Biochim. Biophys. Acta, Bioenerg., 2004, 1657, 82-104.

26 M. Ameloot, J. M. Beechem and L. Brand, Chem. Phys. Lett., 1986, 129, 211-219.

27 J. M. Beechem, M. Ameloot and L. Brand, Chem. Phys. Lett., 1985, 120, 466-472.

28 D. D. S. Fung, L. Qiao, W. C. H. Choy, C. Wang, W. E. I. Sha, F. Xie and S. He, J. Mater. Chem., 2011, 21, 16349-16356.

29 A. Rao, M. Wilson, S. Albert-Seifried, R. Di Pietro and R. Friend, Phys. Rev. B: Condens. Matter Mater. Phys., 2011, 84, 195411.
30 J. Malen, K. Baheti, T. Tong, Y. Zhao, J. Hudgings and A. Majumdar, J. Heat Transfer, 2011, 133, 081601.

31 M. M. Rojo, J. Martin, S. Grauby, T. Borca-Tasciuc, S. Dilhaire and M. Martin-Gonzalez, Nanoscale, 2014, 6, 7858-7865.

32 G. S. Kanner, Z. V. Vardeny and B. C. Hess, Phys. Rev. B: Condens. Matter Mater. Phys., 1990, 42, 5403-5406.

33 J. M. Beechem, M. Ameloot and L. Brand, Anal. Instrum., 1985, 14, 379-402.

34 E. Engel, K. Leo and M. Hoffmann, Chem. Phys., 2006, 325, 170-177.

35 O. Kühn and S. Lochbrunner, Semicond. Semimetals, 2011, 85, 47-81.

36 H. Marciniak, X.-Q. Li, F. Wurthner and S. Lochbrunner, J. Phys. Chem. A, 2011, 115, 648-654.

37 G. Rumbles, I. Samuel, L. Magnani, K. Murray, A. DeMello, B. Crystall, S. Moratti, B. Stone, A. Holmes and R. Friend, Synth. Met., 1996, 76, 47-51.

38 J. Szeremeta, M. Nyk and M. Samoc, Opt. Mater., 2014, 37, 688-694.

39 P. Toman, M. Mensik, W. Bartkowiak and J. Pfleger, Phys. Chem. Chem. Phys., 2017, 19, 7760-7771.

40 Y. Tamai, Y. Matsuura, H. Ohkita, H. Benten and S. Ito, J. Phys. Chem. Lett., 2014, 5, 399-403. 